\title{
Scaphoid tubercle tenderness: a better indicator of scaphoid fractures?
}

\author{
P. FREELAND
}

Department of Accident and Emergency, The Ulster Hospital, Dundonald, Northern Ireland

\section{SUMMARY}

Tenderness in the anatomical snuff box (ASB) has long been accepted as an indicator of a possible scaphoid fracture. The longitudinal axis of the scaphoid lies in an anteroposterior plane, perpendicular to the remainder of the carpal bones, in the radially deviated wrist. The scaphoid tubercle can therefore be easily and accurately palpated on the palmar aspect of the radially deviated wrist.

This study was devised to determine if tenderness over the scaphoid tubercle (ST was superior to ASB tenderness in identifying definite fractures.

Over a 10 -month period 246 patients were seen who were suspected of having scaphoid injury. Thirty $(12 \%)$ were eventually proven to have definite fracture. Tenderness in the ASB had a sensitivity rate of $90 \%$ (95\% confidence limits $80-100 \%$ ) and specificity of $40 \%(33-47 \%)$ as an indicator of a definite fracture. ST tenderness had a sensitivity of $87 \%(75-99 \%)$ and specificity of $57 \%(50-64 \%)$. There is therefore no significant difference in the sensitivity between ASB and ST tenderness but ST tenderness is significantly more specific. However, the presence of either ASB or ST tenderness should be used in order to identify all fractures. Patients with neither ASB nor ST tenderness require symptomatic treatment only.

\section{INTRODUCTION}

A large number of patients are seen in Accident \& Emergency (A \& E) departments with a history of wrist trauma. Tenderness in the anatomical snuff box (ASB) has long been accepted as an indicator of a possible fracture of the scaphoid bone. Many patients without fractures are tender in the ASB, as a branch of the radial nerve crosses the floor of the ASB, and pressure on this nerve may in itself be painful. In addition, scaphoid

Correspondence: Mr P. Freeland, Accident and Emergency Department, Bangour General Hospital, West Lothian EH54 6LR, Scotland. 
fractures are often difficult to identify on radiographs taken soon after injury, even if specialized views are employed (Carver \& Barrington, 1985).

As the consequences of delayed diagnosis and treatment of scaphoid fractures are often serious (Mussbichler, 1961; Haverling \& Sylven, 1978), it has been accepted practice to treat all patients with ASB tenderness in a plaster of Paris cast for 10-14 days before repeating the scaphoid radiography. This involves subjecting many patients with a simple wrist sprain to the inconvenience of a scaphoid cast and further hospital visits.

It can be shown radiographically that while the longitudinal axis of the scaphoid lies in the same place as the remainder of the carpal bones in the ulnar deviated wrist, it rotates to lie in an almost anteroposterior direction when the wrist is radially deviated (Sarrafian et al., 1977) (Fig. $1 \& 2$ ). This allows the scaphoid tubercle to be easily and accurately palpated as a definite 'bump' on the palmar aspect of the wrist when radially deviated.

A prospective study was devised to determine if tenderness over the $S T$ was superior to ASB tenderness in identifying patients with a definite fracture.

\section{METHODS}

All the A \& E doctors were instructed how to localize the ST on the palmar aspect of the radially deviated wrist.

All patients in whom the A \& E doctor suspected a scaphoid injury enough to examine for scaphoid tenderness were included in the study. The presence or absence of tenderness over the ST, in addition to the ASB, was recorded in the A \& E notes before the $\mathrm{X}$-rays were performed. Patients with a definite radiological fracture were managed in the usual way. All patients with ST or ASB tenderness but normal X-rays were immobilized in a scaphoid cast for 14 days before repeating the $\mathrm{X}$-rays.

All X-rays were reported by independent radiologists who were unaware of the details of the study.

Once a final decision had been made about the presence or absence of a fracture, the findings of ASB or ST tenderness were correlated with the radiographic diagnosis. Sensitivity and specificity rates were calculated for ASB and ST tenderness as clinical indicators of a definite scaphoid fracture.

\section{RESULTS}

In the 10-month period from 1 August 1986 to 31 May 1987, 246 patients presented with a history of wrist trauma in whom the A \& E doctor suspected a scaphoid injury. Thirty patients $(12 \%)$ were eventually proven to have a definite scaphoid fracture. Of those with definite fractures $15(50 \%)$ were male and their ages ranged from 10 to 65 with a mean of 26 years. Twenty $(67 \%)$ fractures were diagnosed on the initial X-rays and $10(33 \%)$ on subsequent X-rays taken after 14 days. The findings of ASB and ST tenderness, together with sensitivity and specificity rates, are shown in Table 1. 


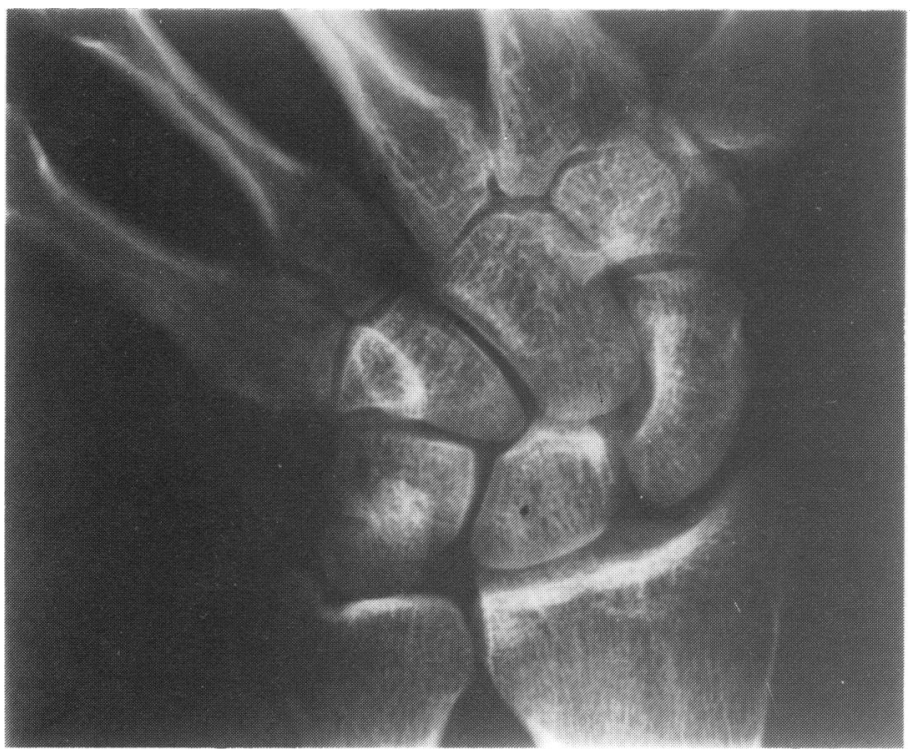

Fig. 1 Ulnar deviated wrist

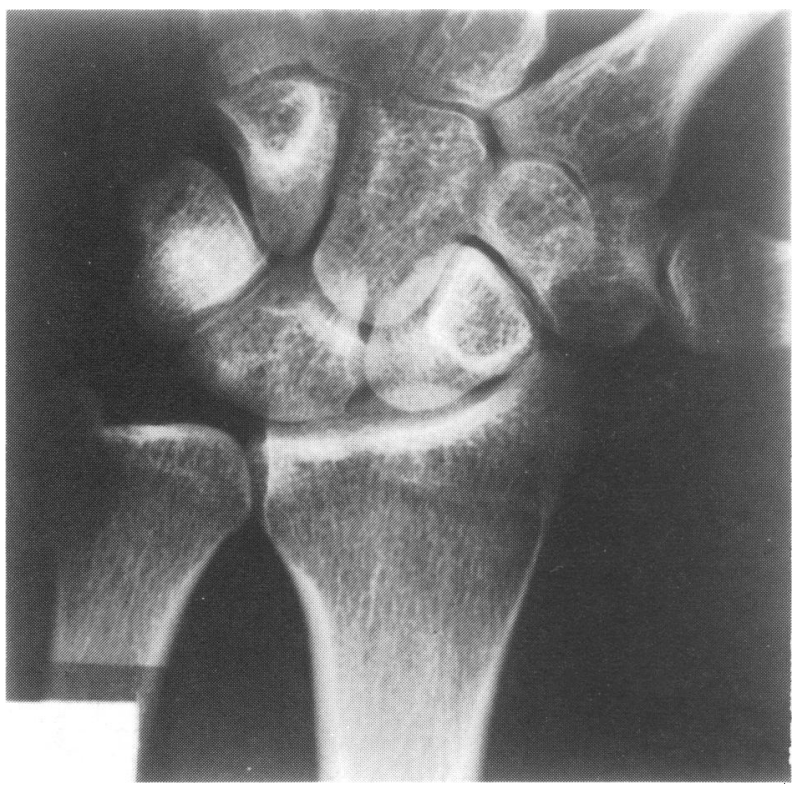

Fig. 2 Radially deviated wrist 
Table 1 ASB and ST tenderness in patients suspected of having a scaphoid injury

\begin{tabular}{lcc}
\hline & Fracture & No fracture \\
\hline ASB tenderness only & 4 & 37 \\
ST tenderness only & 3 & 1 \\
ASB and ST tenderness & 23 & 92 \\
No ASB or ST tenderness & 0 & 86 \\
Totals & 30 & 216 \\
\hline
\end{tabular}

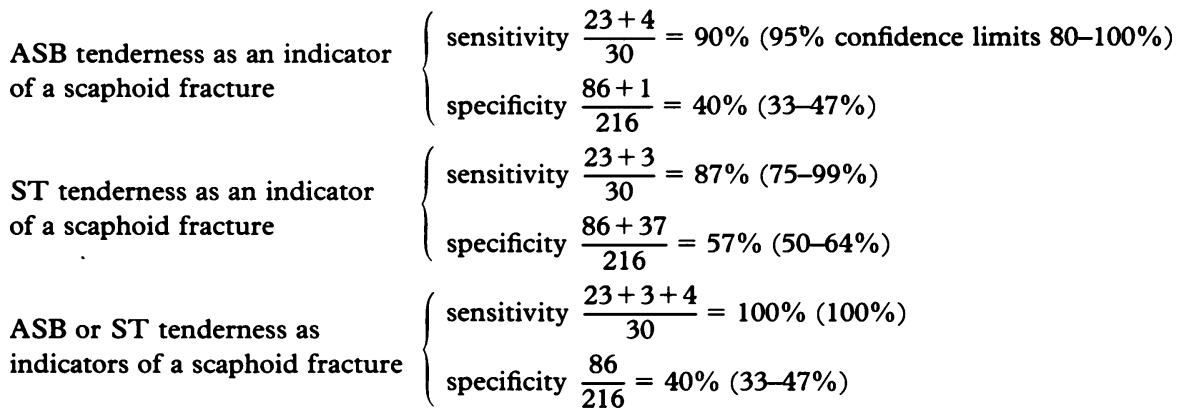

\section{DISCUSSION}

This study demonstrates that although many patients are suspected of having a scaphoid fracture only $12 \%$ are eventually proven. Although described as an injury of the young adult male, our patients covered a wide age distribution with the sexes equally represented. The classically accepted sign of ASB tenderness has a sensitivity rate of $90 \%$ (95\% confidence limits $80-100 \%)$ and a specificity of $40 \%(33-47 \%)$. ST tenderness has a sensitivity of $87 \%(75-99 \%)$ and a specificity of $57 \%(50-64 \%)$. There is, therefore, no significant difference in the sensitivity between ASB and ST tenderness, but ST tenderness is a significantly more specific indicator of a definite scaphoid fracture.

It was interesting to note that the three patients with fractures 'missed' by ASB tenderness were 'picked up' because of their ST tenderness. Similarly, the four fractures missed by ST tenderness had ASB tenderness.

The study was originally devised to determine if ST tenderness could replace ASB tenderness as a more useful indicator of definite scaphoid fractures. Using ST tenderness alone, the number of patients unnecessarily immobilized in a plaster of Paris cast would indeed be significantly reduced. However, the study has unexpectedly shown that using ASB or ST tenderness alone a small number of fractures will be completely missed. In order to avoid missing any fractures therefore, the presence of either ASB or ST tenderness must be used. This would give a specificity rate of $40 \%$, similar to that for ASB tenderness alone, but with a sensitivity of $100 \%$. Patients with neither ASB nor ST tenderness require symptomatic treatment only. 
In conclusion the classically accepted sign of ASB tenderness fails to identify a few patients with a definite scaphoid fracture and has a low specificity. ST tenderness also fails to identify a few fractures but is more specific. The presence of either ASB or PS tenderness should be used in order to identify all fractures.

\section{ACKNOWLEDGEMENTS}

I should like to thank Mr J. Shaw, Mrs A. Ledgerwood, Mrs V. Hastings, the medical, nursing and clerical staff of the A \& E department of the Ulster Hospital, Dundonald, $\overrightarrow{0}$ for their help with this study.

\section{REFERENCES}

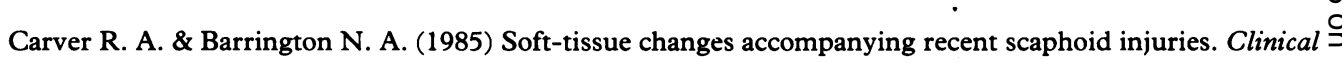
Radiology 36, 423-5.

Haverling M. \& Sylven M. (1978) Soft-tissue abnormalities at fracture of the scaphoid. Data Radiologica Diagnosis 19, 497-501.

Mussbichler H. (1961) Injuries of the carpal scaphoid in children. Data Radiologica Diagnosis 56, 361-8.

Sarrafian S. K., Melamed J. L. \& Goshgarion G. M. (1977) Study of wrist motion in flexion and extension. Clinical Orthopaedics 125, 153-5. 